TRANSACTIONS OF THE

AMERICAN MATHEMATICAL SOCIETY

Volume 363, Number 11, November 2011, Pages 5773-5788

S 0002-9947(2011)05225-6

Article electronically published on May 24, 2011

\title{
ON THE REAL MULTIDIMENSIONAL RATIONAL $K$-MOMENT PROBLEM
}

\author{
JAKA CIMPRIČ, MURRAY MARSHALL, AND TIM NETZER
}

\begin{abstract}
We present a solution to the real multidimensional rational $K$ moment problem, where $K$ is defined by finitely many polynomial inequalities. More precisely, let $S$ be a finite set of real polynomials in $\underline{X}=\left(X_{1}, \ldots, X_{n}\right)$ such that the corresponding basic closed semialgebraic set $K_{S}$ is nonempty. Let $E=D^{-1} \mathbb{R}[\underline{X}]$ be a localization of the real polynomial algebra and let $T_{S}^{E}$ be the preordering on $E$ generated by $S$. We show that every linear functional $L$ on $E$ such that $L\left(T_{S}^{E}\right) \geq 0$ is represented by a positive measure $\mu$ on a certain subset of $K_{S}$, provided $D$ contains an element that grows fast enough on $K_{S}$.
\end{abstract}

\section{INTRODUCTION}

The moment problem for a commutative unital $\mathbb{R}$-algebra $E$ asks to characterize real positive linear functionals on $E$ which can be represented as integrals over measures on an appropriate representation space of $E$. If supports of the measures are required to lie in a prescribed subset $K$ of the representation space, then we talk about the $K$-moment problem on $E$.

A solution to the $K$-moment problem on $\mathbb{R}[\underline{X}]=\mathbb{R}\left[X_{1}, \ldots, X_{n}\right]$ for a compact basic closed semialgebraic set $K$ was given by K. Schmüdgen in [21]. The aim of this paper is to extend his result, both in the compact and noncompact case, to localizations of the polynomial algebra, i.e. to algebras of the form $E=D^{-1} \mathbb{R}[\underline{X}]$, where $D$ is a multiplicative set. The case $n=1, D$ generated by $X_{1}-\alpha$ for countably many real $\alpha$, and $K$ a compact basic closed semialgebraic set, has already been accomplished by J. D. Chandler in 8. Several papers deal with the case $n=1$, $D$ generated by $X_{1}-\alpha$ for countably many real $\alpha$ and $K=\mathbb{R}$; see [6, [7] for surveys. In this case the existence of the solution is rather trivial and the emphasis is on the uniqueness of solutions. The multidimensional case with $D$ generated by $1+\sum_{i=1}^{n} X_{i}^{2}+\sum_{i=1}^{m} g_{i}^{2}$, where $g_{1} \geq 0, \ldots, g_{m} \geq 0$ are the defining relations of $K$, has been achieved by M. Putinar and F.-H. Vasilescu in [16].

To describe our main results we need some terminology. For a finite subset $S=\left\{g_{1}, \ldots, g_{m}\right\}$ of $\mathbb{R}[\underline{X}]$, write $K_{S}=\left\{a \in \mathbb{R}^{n} \mid g_{1}(a) \geq 0, \ldots, g_{m}(a) \geq 0\right\}$ and $T_{S}^{E}$ for the set of all finite sums of elements of the form $e^{2} g_{1}^{\nu_{1}} \cdots g_{m}^{\nu_{m}}$ where $e \in E=D^{-1} \mathbb{R}[\underline{X}]$ and $\nu_{1}, \ldots, \nu_{m} \in\{0,1\}$. We say that a rational function $R \in E$ is $\succeq 0$ on a set $\mathcal{X} \subseteq \mathbb{R}^{n}$ if there exist $f \in \mathbb{R}[\underline{X}]$ and $d \in D$ such that $R=\frac{f}{d}$ and $f d \geq 0$ on $\mathcal{X}$. We write $\mathcal{Z}(d)$ for the set of real zeros of a polynomial $d$.

Received by the editors July 30, 2008 and, in revised form, October 9, 2009.

2010 Mathematics Subject Classification. Primary 44A60, 14 P99.

Key words and phrases. Moment problem, positive polynomials, sums of squares.

(C)2011 American Mathematical Society 
Our main results can be summarized as follows:

Theorem. Let $S$ be a finite subset of $\mathbb{R}[\underline{X}], D$ a multiplicative subset of $\mathbb{R}[\underline{X}]$ such that $1 \in D, 0 \notin D$, and let $R$ be an element of $E=D^{-1} \mathbb{R}[\underline{X}]$. Assume there exists an element $p \in D$ such that $p \geq 1$ on $K_{S}$ and $k p \geq \sum_{i=1}^{n} X_{i}^{2}$ on $K_{S}$ for some integer $k \geq 1$. Then:

(1) $R$ belongs to the closure of $T_{S}^{E}$ in the finest locally convex topology on $E$ if and only if $R \succeq 0$ on $K_{S} \backslash \bigcup_{d \in D} \mathcal{Z}(d)$.

(2) $R$ belongs to the closure of $T_{S}^{E}$ in the topology of finitely open sets on $E$ if and only if $R \succeq 0$ on $K_{S}$.

(3) For every linear functional $L$ on $E$ such that $L\left(T_{S}^{E}\right) \geq 0$, there exists a measure $\mu$ on $\overline{K_{S} \backslash \bigcup_{d \in D} \mathcal{Z}(d)}$ such that

$$
L\left(\frac{f}{d}\right)=\int \frac{f}{d} d \mu \quad \text { for every } \quad \frac{f}{d} \in E .
$$

See Theorems 1, 2] and 4. These results carry over to localizations of an arbitrary finitely generated $\mathbb{R}$-algebra; see Theorem 5 . Assertion (3) of the Theorem solves the moment problem on $E$. For the proof of assertion (3) we use assertion (1) together with a certain rational version of the Riesz-Haviland Theorem which is a generalization of [11, Theorem 3.2.2] and [24, Theorem 3.2]; see Theorem 3,

The conditions on the polynomial $p$ (that $p \geq 1$ on $K_{S}$ and $k p \geq \sum_{i=1}^{n} X_{i}^{2}$ on $K_{S}$ for some integer $k \geq 1$ ) are paraphrased by saying that " $p$ grows fast enough on $K_{S}$ ". See also [11, Theorem 6.2.3] and [22, Theorem 5.1]. One can always take $p=1+\sum_{i=1}^{n} X_{i}^{2}$ (provided, of course, that $1+\sum_{i=1}^{n} X_{i}^{2} \in D$ ). If $K_{S}$ is compact one can take $p=1$, so, in this case, the only assumptions on the multiplicative set $D$ are the trivial ones $1 \in D, 0 \notin D$.

\section{Preorderings AND THE Finest LOCALly CONVEX TOPOLOGY}

Definition. Let $E$ be an $\mathbb{R}$-vector space. A set $U \subseteq E$ is called absorbent if for every $x \in E$ there exists $\lambda>0$ such that $x \in \lambda U$. $U$ is called symmetric if $\lambda U \subseteq U$ for all $\lambda \in[-1,1]$.

The set of all convex, absorbent and symmetric subsets of $E$ forms a zero neighborhood base of a vector space topology on $E$ (see [5, II.25]). It is called the finest locally convex topology on $E$, and the collection of all open sets is denoted by $\mathcal{T}_{\omega}$.

The following are well known:

(T1) $\left(E, \mathcal{T}_{\omega}\right)$ is a topological vector space, i.e. addition and scalar multiplication are continuous. Moreover, $\left(E, \mathcal{T}_{\omega}\right)$ is Hausdorff.

(T2) Every linear mapping from $\left(E, \mathcal{T}_{\omega}\right)$ to a vector space with any locally convex topology is continuous. In particular, all functionals on $\left(E, \mathcal{T}_{\omega}\right)$ are continuous.

These properties are proved in [5, II.26].

A subset $T$ of a commutative ring $E$ with 1 is a preordering if it is closed under addition and multiplication and if it contains the set $E^{2}=\left\{a^{2} \mid a \in E\right\}$. As usual, $\sum E^{2}$ denotes the preordering of $E$ consisting of sums of squares.

Lemma 1. For any preordering $T$ on any commutative $\mathbb{R}$-algebra $E$, its closure in $\mathcal{T}_{\omega}$ is also a preordering. 
Proof. Since $T \subseteq \bar{T}, E^{2} \subseteq \bar{T}$. Since the addition on $E$ is continuous, it follows that $\bar{T}$ is closed under addition. We can't use the same argument for multiplication because by [25. p. 734, Theorem 2] the multiplication on $E=\mathbb{C}(X)$ is not continuous. However, for every $f \in E$, the mapping $\phi_{f}: E \rightarrow E, \phi_{f}(h)=f h$ is linear, and hence continuous by (T2). So, for every $f \in T$,

$$
\phi_{f}(\bar{T}) \subseteq \overline{\phi_{f}(T)} \subseteq \bar{T}
$$

holds, as $\phi_{f}(T) \subseteq T$. It follows that for every $g \in \bar{T}$,

$$
\phi_{g}(\bar{T}) \subseteq \overline{\phi_{g}(T)} \subseteq \bar{T}
$$

as $\phi_{g}(T) \subseteq \bar{T}$, by the above consideration. So for any $g, h \in \bar{T}, g \cdot h=\phi_{g}(h) \in \bar{T}$.

Remark. Note that every preordering $T$ on an $\mathbb{R}$-algebra $E$ is a convex cone, so by (T2) and the Separation Theorem for convex sets (e.g. [5, II.39, Corollary 5]), an element $x$ belongs to $\bar{T}$ if and only if $L(x) \geq 0$ for every linear functional $L$ on $E$ such that $L(T) \geq 0$. It follows that for all elements $a, b \in E$ such that $a+\epsilon b \in T$ for every $\epsilon>0$, we have that $a \in \bar{T}$.

From now on, we are mostly interested in localizations of the real polynomial algebra $\mathbb{R}[\underline{X}]$ in $n$ variables $\underline{X}=\left(X_{1}, \ldots, X_{n}\right)$. So for a multiplicative set $D \subseteq$ $\mathbb{R}[\underline{X}] \backslash\{0\}$ containing 1 , we examine $E=D^{-1} \mathbb{R}[\underline{X}]$.

Remark. While the vector space dimension of $\mathbb{R}[\underline{X}]$ is countable, the dimension of $E$ can be uncountable. For example, if $D$ is the set of polynomials without real zeros, then the uncountable family $\left\{\frac{1}{X_{1}^{2}+c^{2}} \mid c \in \mathbb{R}^{\neq 0}\right\} \subseteq D^{-1} \mathbb{R}[\underline{X}]$ is linearly independent.

For a set $\mathcal{X} \subseteq \mathbb{R}^{n}$, an arbitrary function $\phi: \mathcal{X} \rightarrow \mathbb{R}$ and an element $R \in E=$ $D^{-1} \mathbb{R}[\underline{X}]$, we say

$$
R \succeq \phi \text { on } \mathcal{X}
$$

if there exist $f \in \mathbb{R}[\underline{X}]$ and $d \in D$ such that $R=\frac{f}{d}$ and $(f-d \phi) d \geq 0$ on $\mathcal{X}$. This just means that $R$ has a representation $\frac{f}{d}, f \in \mathbb{R}[\underline{X}], d \in D$, where the function $\frac{f}{d}$ is $\geq \phi$ pointwise on $\mathcal{X}$, wherever it is defined. The set

$$
\operatorname{Pos}^{E}(\mathcal{X})=\{R \in E \mid R \succeq 0 \text { on } \mathcal{X}\}
$$

is a preordering in $E$. We also write

$$
\operatorname{Pos}(\mathcal{X})=\{f \in \mathbb{R}[\underline{X}] \mid f \geq 0 \text { on } \mathcal{X}\}
$$

for the preordering of all polynomials nonnegative on $\mathcal{X}$ in the usual sense. Note that $-1 \notin \operatorname{Pos}(\mathcal{X})$ unless $\mathcal{X}$ is empty. On the other hand, $-1 \in \operatorname{Pos}^{E}(\mathcal{X})$ if $\mathcal{X} \subseteq \mathcal{Z}(d)$ for some $d \in D$. However, if $\mathcal{X} \backslash \mathcal{Z}(d)$ is dense in $\mathcal{X}$ for every $d \in D$, such phenomena cannot occur.

Lemma 2. For every element $R \in E=D^{-1} \mathbb{R}[\underline{X}]$ and every set $\mathcal{X} \subseteq \mathbb{R}^{n}$ such that $\mathcal{X} \backslash \mathcal{Z}(d)$ is dense in $\mathcal{X}$ for every $d \in D$, the following are equivalent:

(1) $R \in \operatorname{Pos}^{E}(\mathcal{X})$,

(2) for every representation $R=\frac{f}{d}$ with $f \in \mathbb{R}[\underline{X}]$ and $d \in D$, we have that $f d \in \operatorname{Pos}(\mathcal{X})$

(3) if we consider $R$ as an element of $\mathbb{R}(\underline{X})$ and write $R=\frac{a}{b}$ where $a, b \in \mathbb{R}[\underline{X}]$, $b \neq 0$ and $\operatorname{gcd}(a, b)=1$, then $a b \in \operatorname{Pos}(\mathcal{X})$.

In particular, for all $f \in \mathbb{R}[\underline{X}], \frac{f}{1} \in \operatorname{Pos}^{E}(\mathcal{X})$ if and only if $f \in \operatorname{Pos}(\mathcal{X})$. 
Proof. Suppose that $R=\frac{f}{d}=\frac{a}{b}$, where $a, b \in \mathbb{R}[\underline{X}], b \neq 0$, satisfy $\operatorname{gcd}(a, b)=1$ and $f \in \mathbb{R}[\underline{X}], d \in D$. Then there exists $u \in \mathbb{R}[\underline{X}] \backslash\{0\}$ such that $f=u a$ and $d=u b$. Clearly, $f d=a b u^{2} \in \operatorname{Pos}(\mathcal{X})$ if and only if $a b \in \operatorname{Pos}(\mathcal{X} \backslash \mathcal{Z}(b u))$. By the assumption on $\mathcal{X}$, this is equivalent to $a b \in \operatorname{Pos}(\mathcal{X})$. Therefore, (1), (2) and (3) are equivalent. The last claim is a special case of (3).

For a finite subset $S=\left\{g_{1}, \ldots, g_{m}\right\}$ of $\mathbb{R}[\underline{X}]$ let

$$
K_{S}=\left\{a \in \mathbb{R}^{n} \mid g_{1}(a) \geq 0, \ldots, g_{m}(a) \geq 0\right\}
$$

be the basic closed semialgebraic set defined by $S$. Let

$$
T_{S}=\left\{\sum_{\nu \in\{0,1\}^{m}} s_{\nu} g_{1}^{\nu_{1}} \cdots g_{m}^{\nu_{m}} \mid s_{\nu} \in \sum \mathbb{R}[\underline{X}]^{2}\right\}
$$

and

$$
T_{S}^{E}=\left\{\sum_{\nu \in\{0,1\}^{m}} s_{\nu} g_{1}^{\nu_{1}} \cdots g_{m}^{\nu_{m}} \mid s_{\nu} \in \sum E^{2}\right\}
$$

be the preorderings generated by $S$ in $\mathbb{R}[\underline{X}]$ and $E=D^{-1} \mathbb{R}[\underline{X}]$, respectively. We always assume $K_{S} \neq \emptyset$, so $-1 \notin T_{S}$. If $K_{S}$ is not contained in any $\mathcal{Z}(d), d \in D$, then $-1 \notin T_{S}^{E}$ also.

The following is our main result:

Theorem 1. Let $D \subseteq \mathbb{R}[\underline{X}] \backslash\{0\}$ be a multiplicative set containing 1 and $S$ a finite subset of $\mathbb{R}[\underline{X}]$. Suppose there is some $p \in D$ such that $p \geq 1$ on $K_{S}$ and $k p-\sum_{i=1}^{n} X_{i}^{2} \geq 0$ on $K_{S}$ for some $k \geq 1$. Then

$$
\overline{T_{S}^{E}}=\operatorname{Pos}^{E}\left(K_{S} \backslash \bigcup_{d \in D} \mathcal{Z}(d)\right)
$$

holds in $E=D^{-1} \mathbb{R}[\underline{X}]$.

The set $\mathcal{X}=K_{S} \backslash \bigcup_{d \in D} \mathcal{Z}(d)$ satisfies the assumptions of Lemma 2, which gives two characterizations of $\operatorname{Pos}^{E}(\mathcal{X})$. Another characterization is that $R \in \operatorname{Pos}^{E}(\mathcal{X})$ if and only if $\chi(R) \geq 0$ for every unital $\mathbb{R}$-algebra homomorphism $\chi: E \rightarrow \mathbb{R}$ such that $\chi(S) \geq 0$.

We will give the proof of Theorem 1 in Section 3 . In Section 4 we will show that Theorem 1 implies the solution of the real multidimensional rational $K$-moment problem. In Section 5 we will prove a variant of Theorem 1 for the topology of finitely open sets.

Note that in the case where $K_{S}$ is compact, we can always take $p=1$ in the Theorem. In the noncompact case, the polynomial $p=1+\sum_{i=1}^{n} X_{i}^{2}$ can always be used in the application of Theorem 1 as long as it belongs to the multiplicative set $D$. We record an easy corollary of Theorem 1, in which this is the case. Therefore let $\mathbb{R}(\underline{X} ; \mathcal{P})$ be the algebra of real rational functions with (real) poles only in a given set $\mathcal{P} \subseteq \mathbb{R}^{n}$. It is the localization of the polynomial algebra with respect to the set $D$ of nonzero polynomials with real zeros only in $\mathcal{P}$. The set of zeros of elements from $D$ equals $\mathcal{P}$. The polynomial $p=1+\sum_{i=1}^{n} X_{i}^{2}$ belongs to $D$.

Corollary 1. In $E=\mathbb{R}(\underline{X} ; \mathcal{P})=D^{-1} \mathbb{R}[\underline{X}]$ we have for arbitrary finite sets $S \subseteq$ $\mathbb{R}[\underline{X}]$,

$$
\overline{T_{S}^{E}}=\operatorname{Pos}^{E}\left(K_{S} \backslash \mathcal{P}\right) .
$$


In particular,

$$
\begin{aligned}
& \text { In particular, } \\
& \qquad \begin{array}{c}
\overline{\sum \mathbb{R}(\underline{X} ; \mathcal{P})^{2}}=\operatorname{Pos}^{E}\left(\mathbb{R}^{n} \backslash \mathcal{P}\right) \text {. } \\
\text { If } \mathcal{P}=\mathbb{R}^{n} \text {, then } \mathbb{R}(\underline{X} ; \mathcal{P})=\frac{\mathbb{R}(\underline{X}) \text { and } \operatorname{Pos}^{E}\left(\mathbb{R}^{n} \backslash \mathcal{P}\right)=\mathbb{R}(\underline{X}) \text {, so }}{\overline{\sum \mathbb{R}(\underline{X})^{2}}=\mathbb{R}(\underline{X}) .}
\end{array}
\end{aligned}
$$

In particular, there is no positive linear functional on $\mathbb{R}(\underline{X})$.

The last claim of Corollary 1 also follows from [14, 9.7.29].

Remark. Let $L / \mathbb{R}$ be a proper field extension. We claim that there is no nontrivial positive linear functional on $L$. If $L$ is not real, then every element is a sum of squares, so there is clearly no nontrivial positive linear functional. Otherwise, every element $a$ of $L \backslash \mathbb{R}$ is transcendental over $\mathbb{R}$. So $\mathbb{R}(a)$ is isomorphic to $\mathbb{R}(X)$, and by Corollary 1 there is no nontrivial positive linear functional on $\mathbb{R}(a)$.

Remark. When $K_{S}$ is compact and disjoint from $\bigcup_{d \in D} \mathcal{Z}(d)$, we can prove Theorem 11 by the usual analytic trick. Namely, in this case Wörmann's trick implies that $T_{S}^{E}$ is archimedean. (Since Wörmann's trick works only for finitely generated $\mathbb{R}$-algebras, we apply it first to algebras $\mathbb{R}[\underline{X}]_{d}$ and then use the fact that $E=D^{-1} \mathbb{R}[\underline{X}]$ is equal to $\bigcup_{d \in D} \mathbb{R}[\underline{X}]_{d}$.) Then the Kadison-Dubois Representation Theorem implies that the closure of $T_{S}^{E}$ is equal to $\operatorname{Pos}^{E}\left(K_{S}\right)$. We refer the reader to [11] for the statement of Wörmann's trick and the Representation Theorem. We will not pursue this idea further.

\section{The Proof of Theorem 1}

The easy inclusion $\overline{T_{S}^{E}} \subseteq \operatorname{Pos}^{E}\left(K_{S} \backslash \bigcup_{d \in D} \mathcal{Z}(d)\right)$ follows from the obvious fact that evaluation in a point from $K_{S} \backslash \bigcup_{d \in D} \mathcal{Z}(d)$ defines a linear functional $L$ on $E$ such that $L\left(T_{S}^{E}\right) \geq 0$.

The proof of the difficult inclusion $\overline{T_{S}^{E}} \supseteq \operatorname{Pos}^{E}\left(K_{S} \backslash \bigcup_{d \in D} \mathcal{Z}(d)\right)$ will be split into several lemmas.

Lemma 3. Let $D \subseteq \mathbb{R}[\underline{X}] \backslash\{0\}$ be a multiplicative set containing 1 , $K$ a compact set contained in $\mathcal{P}=\bigcup_{d \in D} \mathcal{Z}(d)$, and $c \in \mathbb{R}^{>0}$. Then every neighborhood of zero in $D^{-1} \mathbb{R}[\underline{X}]$ (in the finest locally convex topology) contains an element $R$ such that $R \succeq c \cdot \chi_{K}$ globally, where $\chi_{K}$ is the characteristic function of $K$.

Proof. Let $N$ be a neighborhood of zero in $D^{-1} \mathbb{R}[\underline{X}]$. Without loss of generality assume that $N$ is convex and absorbent. Hence for any $f \in D^{-1} \mathbb{R}[\underline{X}]$ there is a number $\delta(f)>0$ such that $\lambda f \in N$ for all $\lambda \in[-\delta(f), \delta(f)]$.

For every $a \in \bigcup_{d \in D} \mathcal{Z}(d)$ we have $d_{a}(a)=0$ for some $d_{a} \in D$, so that $d_{a}(x)=$ $\left(\nabla d_{a}\right)(a) \cdot(x-a)+o(\|x-a\|)$. Write $c_{a}=\left\|\left(\nabla d_{a}\right)(a)\right\|+1$ and pick $\eta_{a}>0$ such that

$$
\left|d_{a}(x)\right| \leq c_{a}\|x-a\| \text { on } B\left(a, \eta_{a}\right) .
$$

Let $W$ be a cube containing $K$ and let $B_{n}$ be the unit ball in $\mathbb{R}^{n}$. Define

$$
\lambda:=\frac{1}{6}\left(\frac{\operatorname{vol}\left(B_{n}\right)}{c \operatorname{vol}(W)}\right)^{\frac{1}{n}}
$$

and

$$
r_{a}:=\min \left\{\lambda c_{a}^{-2} \delta\left(d_{a}^{-2 n}\right)^{\frac{1}{n}}, \operatorname{vol}(W)^{\frac{1}{n}}, \eta_{a}\right\}
$$


Therefore, $r_{a}>0$ and

$$
K \subseteq \bigcup_{a \in K} B\left(a, \frac{r_{a}}{3}\right)
$$

where $B\left(a, \frac{r_{a}}{3}\right)$ denotes the open ball of radius $\frac{r_{a}}{3}$ around $a$. By Wiener's Covering Lemma, 10, Lemma 4.1.1], there are $a_{1}, \ldots, a_{t} \in K$ such that

$$
K \subseteq \bigcup_{i=1}^{t} B\left(a_{i}, r_{a_{i}}\right)
$$

and the $B\left(a_{i}, \frac{r_{a_{i}}}{3}\right)$ are pairwise disjoint.

For every ball $B$ with center in $W$ and with radius less than or equal to half the side length of $W$, we have

$$
\operatorname{vol}(B \cap W) \geq \frac{1}{2^{n}} \operatorname{vol}(B),
$$

since $\operatorname{vol}(B \cap W)$ is minimal if the center of $B$ lies in one of the corners of $W$. In particular, this is true for $B=B\left(a_{i}, \frac{r_{a_{i}}}{3}\right), i=1, \ldots, t$, by (3). Since $B\left(a_{i}, \frac{r_{a_{i}}}{3}\right)$ are pairwise disjoint, it follows that

$$
\sum_{i=1}^{t} \frac{1}{2^{n}} \operatorname{vol}\left(B\left(a_{i}, \frac{r_{a_{i}}}{3}\right)\right) \leq \operatorname{vol}(W) .
$$

Combining this with the volume formula

$$
\operatorname{vol}\left(B\left(a_{i}, \frac{r_{a_{i}}}{3}\right)\right)=\left(\frac{r_{a_{i}}}{3}\right)^{n} \operatorname{vol}\left(B_{n}\right),
$$

we get

$$
\sum_{i=1}^{t} r_{a_{i}}^{n} \leq \frac{6^{n} \operatorname{vol}(W)}{\operatorname{vol}\left(B_{n}\right)}=\frac{1}{\lambda^{n} c}
$$

where the last equality follows from (2). Now define

$$
R:=\frac{1}{\lambda^{n} \sum_{i=1}^{t} r_{a_{i}}^{n}} \cdot \sum_{i=1}^{t} \frac{r_{a_{i}}^{2 n} c_{a_{i}}^{2 n}}{d_{a_{i}}^{2 n}}=\sum_{i=1}^{t} \frac{r_{a_{i}}^{n}}{\sum_{j=1}^{t} r_{a_{j}}^{n}} \cdot \frac{r_{a_{i}}^{n} c_{a_{i}}^{2 n}}{\lambda^{n} d_{a_{i}}^{2 n}}
$$

Clearly $R \in D^{-1} \mathbb{R}[\underline{X}]$ and, as $\frac{r_{a_{i}}^{n} c_{a_{i}}^{2 n}}{\lambda^{n}} \leq \delta\left(d_{a_{i}}^{-2 n}\right)$ by (3), each $\frac{r_{a_{i}}^{n} c_{a_{i}}^{2 n}}{\lambda^{n} d_{a_{i}}^{2 n}}$ lies in $N$. So $R$, as a convex combination of such elements, also lies in $N$. By (11) and (3), $\frac{r_{a_{i}}^{2 n} c_{a_{i}}^{2 n}}{d_{a_{i}}^{2 n}} \succeq \chi_{B\left(a_{i}, r_{a_{i}}\right)}$ globally, so one checks that

$$
\sum_{i=1}^{t} \frac{r_{a_{i}}^{2 n} c_{a_{i}}^{2 n}}{d_{a_{i}}^{2 n}} \succeq \chi_{K}
$$

Therefore, $R \succeq c \cdot \chi_{K}$ by (4).

Lemma 4. Let $D \subseteq \mathbb{R}[\underline{X}] \backslash\{0\}$ be a multiplicative set containing 1 and $S \subseteq \mathbb{R}[\underline{X}]$ finite. Assume there is some $p \in D$ such that $p \geq 1$ on $K_{S}$, and $k p-\sum_{i=1}^{n} X_{i}^{2} \geq 0$ on $K_{S}$ for some $k \geq 1$. Let $K$ be a compact subset of $\mathcal{P}=\bigcup_{d \in D} \mathcal{Z}(d)$. Then every $f \in \mathbb{R}[\underline{X}]$ which is nonnegative on $K_{S} \backslash K$ belongs to $\overline{T_{S}^{E}}$ in $E=D^{-1} \mathbb{R}[\underline{X}]$. 
Proof. Take any neighborhood of zero $N$ in $E$. Let $m$ be the minimum of $f$ on $K_{S} \cap K$. By Lemma 3, there exists $R \in N$ such that $R \succeq-m \cdot \chi_{K}$. Hence we find a representation $f+R=\frac{u}{v}$ with $u v \geq 0$ on $K_{S}$. By [22, Theorem 5.1] 1 the polynomial $u v$ belongs to the closure of the preordering generated by $S$ in $\mathbb{R}\left[\underline{X}, \frac{1}{p}\right]$, so also to the closure of $T_{S}^{E}$. Therefore, $f+R=\left(\frac{1}{v}\right)^{2} u v \in(f+N) \cap \overline{T_{S}^{E}}$. Hence $f \in \overline{T_{S}^{E}}$, as $N$ was arbitrary.

In the next lemma we eliminate the compactness assumption:

Lemma 5. Lemma 4 also holds when $K=\mathcal{P}$.

Proof. For every $\epsilon>0, f+\epsilon p^{\operatorname{deg}(f)+1}$ is nonnegative on $K_{S} \backslash B_{\epsilon}$ for a closed ball $B_{\epsilon}$ around 0. Define

$$
K_{\epsilon}:=\left\{a \in K_{S} \cap B_{\epsilon} \mid f(x) \leq-\epsilon\right\} .
$$

$K_{\epsilon}$ is a compact subset of $\mathcal{P}=\bigcup_{d \in D} \mathcal{Z}(d)$, and $f+\epsilon+\epsilon p^{\operatorname{deg}(f)+1}$ is nonnegative on $K_{S} \backslash K_{\epsilon}$. By Lemma 4, $f+\epsilon+\epsilon p^{\operatorname{deg}(f)+1}$ belongs to the closure of $T_{S}^{E}$ in $E=D^{-1} \mathbb{R}[\underline{X}]$. As this is true for all $\epsilon>0, f$ belongs to $\overline{T_{S}^{E}}$.

Now we give the

Proof of Theorem 1. Take $\frac{f}{d} \in E$ with $f d \in \operatorname{Pos}\left(K_{S} \backslash \mathcal{P}\right)$. Then apply Lemma 5 to obtain $f d \in \overline{T_{S}^{E}}$ and multiply with $\left(\frac{1}{d}\right)^{2}$.

\section{RATional MOMENT PROBlems}

The aim of this section is to prove the following existence result for the multidimensional rational $K$-moment problem, which in the one-dimensional compact case extends [8, Theorem 5].

Theorem 2. Let $D \subseteq \mathbb{R}[\underline{X}] \backslash\{0\}$ be a multiplicative set containing 1 and let $S \subseteq \mathbb{R}[\underline{X}]$ be finite. Assume there is some $p \in D$ such that $p \geq 1$ on $K_{S}$, and $k p-\sum_{i=1}^{n} X_{i}^{2} \geq 0$ on $K_{S}$ for some $k \geq 1$.

Then, for every linear functional $L$ on $E=D^{-1} \mathbb{R}[\underline{X}]$ such that $L\left(T_{S}^{E}\right) \geq 0$, there exists a measure $\mu$ on $\overline{K_{S} \backslash \bigcup_{d \in D} \mathcal{Z}(d)}$ such that

$$
L\left(\frac{f}{d}\right)=\int \frac{f}{d} d \mu
$$

for every $\frac{f}{d} \in D^{-1} \mathbb{R}[\underline{X}]$.

If $K_{S}$ has empty intersection with $\mathcal{P}=\bigcup_{d \in D} \mathcal{Z}(d)$, then Theorem 2 follows from our Theorem 1 and [11, Theorem 3.2.2]. To prove the general case, we need the following generalization of [11, Theorem 3.2.2] (applied to $A=\mathbb{R}[\underline{X}], \mathcal{X}=$ $\overline{K_{S} \backslash \bigcup_{d \in D} \mathcal{Z}(d)}, \hat{a}=\left.a\right|_{\mathcal{X}}$ and $\left.q=\sum X_{i}^{2}\right)$.

Theorem 3 (Rational Haviland's Theorem). Let $A$ be a unital $\mathbb{R}$ algebra, $D$ a multiplicative subset of $A$ containing $1, \mathcal{X}$ a nonempty Hausdorff topological space

\footnotetext{
${ }^{1}$ Note that our assumption on $p$ implies that condition $(*)$ of [22, Theorem 5.1] is satisfied. If $p$ satisfies, instead of $p \geq 1$ on $K_{S}$, the stronger assumption $p-1 \in T_{S}$ (e.g. when $p=1+\sum_{i=1}^{n} X_{i}^{2}$ ), then we can use [11, Theorem 6.2.3] (or [12, Corollary 3.2]), which is a slightly weaker version of 22 Theorem 5.1].
} 
and ${ }^{\wedge}: A \rightarrow \mathcal{C}(\mathcal{X}, \mathbb{R})$ an $\mathbb{R}$-algebra homomorphism. Suppose that:

(1) there exists $q \in A$ such that $\hat{q} \geq 0$ on $\mathcal{X}$ and, for each $k \geq 1$, the set $\mathcal{X}_{k}=\{x \in \mathcal{X} \mid \hat{q}(x) \leq k\}$ is compact,

(2) for every $d \in D$, the zero set $\mathcal{Z}(\hat{d})$ of $\hat{d}$ in $\mathcal{X}$ has empty interior,

(3) every open subset of $\mathcal{X}$ is $\sigma$-compact ( $=$ a countable union of compact sets).

Then, for any linear functional $L: D^{-1} A \rightarrow \mathbb{R}$ satisfying

$$
\forall a \in A, \forall d \in D, \hat{a} \hat{d} \geq 0 \text { on } \mathcal{X} \Rightarrow L(a / d) \geq 0,
$$

there exists a Borel measure $\mu$ on $\mathcal{X}$ such that

$$
\forall a \in A, \forall d \in D, L(a / d)=\int_{\mathcal{X}} \hat{a} / \hat{d} d \mu .
$$

The idea is to follow the proof of [11, Theorem 3.2.2] (or [13, Theorem 3.1]) and use [24, Theorem 3.2] instead of Riesz's Theorem. We start with a few remarks:

R1. Assumption (1) implies that $\mathcal{X}$ is locally compact.

R2. For every $d \in D$, the following assertions are equivalent:

(a) $\mathcal{X} \backslash \mathcal{Z}(\hat{d})$ is dense in $\mathcal{X}$

(b) $\mathcal{Z}(\hat{d})$ has empty interior,

(c) for every $f \in \mathcal{C}(\mathcal{X}, \mathbb{R})$, if $f \hat{d}^{2} \geq 0$ on $\mathcal{X}$, then $f \geq 0$ on $\mathcal{X}$,

(d) for every $f \in \mathcal{C}(\mathcal{X}, \mathbb{R})$, if $f \hat{d} \equiv 0$ on $\mathcal{X}$, then $f \equiv 0$ on $\mathcal{X}$.

Every locally compact Hausdorff space is completely regular, which means that points can be separated from closed sets by continuous functions (Urysohn's Lemma; see $[19,2.12])$, so we have that $(\mathrm{d}) \Rightarrow(\mathrm{b})$. Implications (a) $\Leftrightarrow(\mathrm{b}) \Rightarrow(\mathrm{c}) \Rightarrow(\mathrm{d})$ are clear.

R3. Assumption (2) implies that $0 \notin \hat{D}$, hence $0 \notin D$. Therefore, $D^{-1} A$ and $\hat{D}^{-1} \mathcal{C}(\mathcal{X}, \mathbb{R})$ are nontrivial $\mathbb{R}$-algebras.

R4. The homomorphism ${ }^{\wedge}: A \rightarrow \mathcal{C}(\mathcal{X}, \mathbb{R})$ extends uniquely to a homomorphism $\hat{~}: D^{-1} A \rightarrow \hat{D}^{-1} \mathcal{C}(\mathcal{X}, \mathbb{R})$. Its image is $\widehat{D^{-1} A}=\hat{D}^{-1} \hat{A}$.

R5. $\bar{L}: \hat{D}^{-1} \hat{A} \rightarrow \mathbb{R}$ defined by $\bar{L}(\hat{a} / \hat{d})=L(a / d)$ is well-defined. This follows from the positivity assumption on $L$. (See Claim 1 in the proof of [11, Theorem 3.2.2].)

R6. We can assume that $\hat{d} \geq 0$ on $\mathcal{X}$ for every $d \in D$. Namely, every element $f / \hat{d} \in \hat{D}^{-1} \mathcal{C}(\mathcal{X}, \mathbb{R})$ can be written as $f / \hat{d}=f \hat{d} / \widehat{d}^{2}$ and $\widehat{d}^{2} \geq 0$ on $\mathcal{X}$. The positivity assumption on $L$ then simplifies to: $\forall a \in A, \forall d \in D, \hat{a} \geq 0$ on $\mathcal{X} \Rightarrow L(a / d) \geq 0$.

R7. Let $B=\hat{D}^{-1} \mathcal{C}^{\prime}(\mathcal{X}, \mathbb{R})$, where $\mathcal{C}^{\prime}(\mathcal{X}, \mathbb{R})=\{f \in \mathcal{C}(\mathcal{X}, \mathbb{R})|\exists a \in A:| f \mid \leq$ $\hat{a}$ on $\mathcal{X}\}$. Note that $B$ is a subalgebra of $\hat{D}^{-1} \mathcal{C}(\mathcal{X}, \mathbb{R})$ and $B=\left\{f / \hat{d} \in \hat{D}^{-1} \mathcal{C}(\mathcal{X}, \mathbb{R}) \mid\right.$ $\exists a \in A, s \in D:|f| \hat{s} \leq \hat{a} \hat{d}$ on $\mathcal{X}\}$. Namely, if $|f| \hat{s} \leq \hat{a} \hat{d}$ on $\mathcal{X}$, then $f / \hat{d}=f \hat{s} / \widehat{d s}$ and $f \hat{s} \in \mathcal{C}^{\prime}(\mathcal{X}, \mathbb{R})$. The other inclusion is clear. 
R8. The algebra $B$ obviously contains both $\hat{D}^{-1} \hat{A}$ and $\hat{D}^{-1} \mathcal{C}_{c}(\mathcal{X}, \mathbb{R})$. Here, $\mathcal{C}_{c}(\mathcal{X}, \mathbb{R})$ denotes the algebra of continuous functions with compact support.

Lemma 6. $\bar{L}$ extends to a linear functional $\bar{L}$ on $B=\hat{D}^{-1} \mathcal{C}^{\prime}(\mathcal{X}, \mathbb{R})$ such that $\bar{L}(f / \hat{d}) \geq 0$ for every $d \in D$ and every nonnegative $f \in \mathcal{C}^{\prime}(\mathcal{X}, \mathbb{R})$.

Proof. The proof is similar to the proof of Claim 2 in the proof of [11, Theorem 3.2 .2 . This result is also a special case of a more general extension theorem for positive functionals; see [2, Theorem 2.6.2, p. 69].

Lemma 7 (Rational Riesz's Theorem). For every linear functional $\bar{L}: \hat{D}^{-1} \mathcal{C}_{c}(\mathcal{X}, \mathbb{R})$ $\rightarrow \mathbb{R}$ such that $\bar{L}(f / \hat{d}) \geq 0$ for every nonnegative $f \in \mathcal{C}_{c}(\mathcal{X}, \mathbb{R})$, there exists a unique Borel measure $\mu$ on $\mathcal{X}$ such that $\bar{L}(f / \hat{d})=\int_{\mathcal{X}} f / \hat{d} d \mu$ for every $f / \hat{d} \in \hat{D}^{-1} \mathcal{C}_{c}(\mathcal{X}, \mathbb{R})$.

Proof. (Based on the proof of [24, Theorem 3.2].) For every $s \in D$ define a functional $\bar{L}_{s}: \mathcal{C}_{c}(\mathcal{X}, \mathbb{R}) \rightarrow \mathbb{R}$ by $\bar{L}_{s}(f)=\bar{L}(f / \hat{s})$. Since $\bar{L}_{s}(f) \geq 0$ for every $f \geq 0$, there exists by Riesz's Theorem (see [19, Theorem 2.14]), a unique regular Borel measure $\mu_{s}$ on $\mathcal{X}$ such that $\bar{L}_{s}(f)=\int_{\mathcal{X}} f d \mu_{s}$ for every $f \in \mathcal{C}_{c}(\mathcal{X}, \mathbb{R})$. Write $\mu=\mu_{1}$ and note that

$$
\int_{\mathcal{X}} f d \mu=\int_{\mathcal{X}} f \hat{s} d \mu_{s}
$$

for every $f \in \mathcal{C}_{c}(\mathcal{X}, \mathbb{R})$. By [19, Theorem 1.29], $\hat{s} d \mu_{s}$ is a Borel measure on $\mathcal{X}$. To prove that $\hat{s} d \mu_{s}$ is regular, we apply [19, Theorem 2.18]. (Here we need our assumption (3) and the continuity of $\hat{s}$.) By the uniqueness part of Riesz's Theorem, $\mu=\hat{s} d \mu_{s}$.

Now, we will prove that $\mu_{s}(\mathcal{Z}(\hat{s}))=0$. By a version of Urysohn's Lemma (see [19, Theorem 2.12]), there exists for each integer $k$ a function $u_{k} \in \mathcal{C}_{c}(\mathcal{X}, \mathbb{R})$ such that $0 \leq u_{k} \leq 1$ on $\mathcal{X}$ and $u_{k}=1$ on $\mathcal{Z}(\hat{s}) \cap \mathcal{X}_{k}$. For all integers $i, k$, the function $f_{k, i}=u_{k} \min \{1,1 / i \hat{s}\}$ belongs to $\mathcal{C}_{c}(\mathcal{X}, \mathbb{R}), 0 \leq f_{k, i} \leq 1$ on $\mathcal{X}$ and $f_{k, i}=1$ on $\mathcal{Z}(\hat{s}) \cap \mathcal{X}_{k}$. It follows that

$$
\begin{aligned}
& \mu_{s}\left(\mathcal{Z}(\hat{s}) \cap \mathcal{X}_{k}\right) \leq \int_{\mathcal{X}} f_{k, i} d \mu_{s} \\
& \quad=\bar{L}_{s}\left(f_{k, i}\right)=\bar{L}\left(f_{k, i} / \hat{s}\right) \leq(1 / i) \bar{L}\left(u_{k} / \hat{s}^{2}\right) .
\end{aligned}
$$

Sending $i \rightarrow \infty$, we get $\mu_{s}\left(\mathcal{Z}(\hat{s}) \cap \mathcal{X}_{k}\right)=0$ for every $k$, hence $\mu_{s}(\mathcal{Z}(\hat{s}))=0$. In particular, $\mu(\mathcal{Z}(\hat{s}))=0$ (which is also a consequence of $\mu=\hat{s} d \mu_{s}$ ). Finally, for every $f \in \mathcal{C}_{c}(\mathcal{X}, \mathbb{R})$ and every $s \in D$ we have that

$$
\begin{array}{r}
\int_{\mathcal{X}} f / \hat{s} d \mu=\int_{\mathcal{X} \backslash \mathcal{Z}(\hat{s})} f / \hat{s} d \mu=\int_{\mathcal{X} \backslash \mathcal{Z}(\hat{s})}(f / \hat{s}) \hat{s} d \mu_{s} \\
=\int_{\mathcal{X} \backslash \mathcal{Z}(\hat{s})} f d \mu_{s}=\int_{\mathcal{X}} f d \mu_{s}=\bar{L}_{s}(f)=\bar{L}(f / \hat{s}) .
\end{array}
$$

Lemma 8. For every nonnegative function $f \in \mathcal{C}^{\prime}(\mathcal{X}, \mathbb{R})$, there exists a monotonically increasing sequence of nonnegative functions $f_{i} \in \mathcal{C}_{c}(\mathcal{X}, \mathbb{R})$ such that $\frac{(f+\hat{q})^{2}}{i} \geq f-f_{i} \geq 0$ on $\mathcal{X}$ for every $i$.

This is Claim 3 in the proof of [11, Theorem 3.2.2]. No changes are needed. 
Proof of the Theorem. Let $\bar{L}$ be a linear functional on $\hat{D}^{-1} \hat{A}$ such that $\bar{L}(f / \hat{d}) \geq 0$ for every nonnegative $f \in \hat{A}$ and every $d \in D$. By Lemma [6] we can extend $\bar{L}$ to a linear functional $\bar{L}$ on $\hat{D}^{-1} \mathcal{C}^{\prime}(\mathcal{X}, \mathbb{R})$ such that $\bar{L}(f / \hat{d}) \geq 0$ for every nonnegative $f \in \mathcal{C}^{\prime}(\mathcal{X}, \mathbb{R})$. Lemma 7 gives us a Borel measure $\mu$ on $\mathcal{X}$ such that $\bar{L}(f / \hat{d})=$ $\int_{\mathcal{X}} f / \hat{d} d \mu$ for every $f \in \mathcal{C}_{c}(\mathcal{X}, \mathbb{R})$. Lemma 8 implies that $\bar{L}(f / \hat{d})=\int_{\mathcal{X}} f / \hat{d} d \mu$ for every $f \in \mathcal{C}^{\prime}(\mathcal{X}, \mathbb{R})$. Namely,

$$
\int_{\mathcal{X}} f / \hat{d} d \mu=\lim _{i \rightarrow \infty} \int_{\mathcal{X}} f_{i} / \hat{d} d \mu=\lim _{i \rightarrow \infty} \bar{L}\left(f_{i} / \hat{d}\right)=\bar{L}(f / \hat{d}),
$$

by the Monotone Convergence Theorem, the inequality $\frac{(f+\hat{q})^{2}}{i} \geq f-f_{i} \geq 0$ and the positivity of $\bar{L}$. In particular, $L(a / d)=\bar{L}(\hat{a} / \hat{d})=\int_{\mathcal{X}} \hat{a} / \hat{d} d \mu$ for every $a \in A$ and $d \in D$.

\section{Preorderings And the topology of Finitely open Sets}

Definition. Let $E$ be an $\mathbb{R}$-vector space and $O$ a subset of $E$. We say that $O$ is open in the topology of finitely open sets if for every finite-dimensional vector subspace $U$ of $E, O \cap U$ is open in the usual topology of $U$. Write $\mathcal{T}_{\text {fin }}$ for the set of all open sets.

We recall the following results from $[3]$ :

(F1) If $E$ has countable dimension, then the topology $\mathcal{T}_{\text {fin }}$ is equal to the topology $\mathcal{T}_{\omega}$. If $E$ does not have countable dimension, then the topology $\mathcal{T}_{\text {fin }}$ is strictly finer (= has more open sets) than the topology $\mathcal{T}_{\omega}$. In particular, $\mathcal{T}_{\text {fin }}$ is not a locally convex topology in this case.

(F2) If $E$ does not have countable dimension, then the addition is not continuous in the topology $\mathcal{T}_{\text {fin }}$.

(F3) Let $E$ be an $\mathbb{R}$-vector space and $\mathcal{D}$ the set of all countable-dimensional subspaces of $E$. Then, for every subset $A$ of $E$

$$
\bar{A}=\bigcup_{D \in \mathcal{D}} \overline{A \cap D} .
$$

The following should be well known, but we were unable to find a reference:

(F4) Any linear mapping between any two vector spaces both having the topology of finitely open sets is continuous. In particular, every functional on every vector space with the finitely open topology is continuous.

Proof. Let $L:\left(E, \mathcal{T}_{\text {fin }}\right) \rightarrow\left(E^{\prime}, \mathcal{T}_{\text {fin }}^{\prime}\right)$ be a linear mapping and $O \in \mathcal{T}_{\text {fin }}^{\prime}$. Pick any finite-dimensional subspace $V$ of $E$. Clearly, the subspace $L(V)$ of $E^{\prime}$ is also finitedimensional. By the definition of $\mathcal{T}_{\text {fin }}^{\prime}, O \cap L(V)$ is open in the usual topology of $L(V)$. Every linear mapping between two finite-dimensional vector spaces is continuous in the usual topologies. In particular $\left.L\right|_{V}: V \rightarrow L(V)$ is continuous in the usual topologies of $V$ and $L(V)$. It follows that the set $\left(\left.L\right|_{V}\right)^{-1}(O \cap L(V))$ is open in the usual topology of $V$. Note that $\left(\left.L\right|_{V}\right)^{-1}(O \cap L(V))=L^{-1}(O) \cap V$, hence $L^{-1}(O) \cap V$ is open in the usual topology of $V$. Since $V$ was arbitrary, it follows by the definition of $\mathcal{T}_{\text {fin }}$ that $L^{-1}(O)$ is open. Since $O$ was arbitrary, $L$ is indeed continuous.

Lemma 9. For a preordering $T$ on a commutative $\mathbb{R}$-algebra $E$, its closure in $\mathcal{T}_{\text {fin }}$ is also a preordering. 
Proof. For any $f_{1}, f_{2} \in \bar{T}$, there exist, by (F3), countable-dimensional subspaces $D_{1}, D_{2}$ of $E$ such that $f_{i} \in \overline{T \cap D_{i}}$ for $i=1,2$. The subspace $D=D_{1}+D_{2}$ is also countable-dimensional, and $\overline{T \cap D_{i}} \subseteq \overline{T \cap D}$ for $i=1,2$. It follows that $f_{1}, f_{2} \in \overline{T \cap D}$. Since $D$ is countable-dimensional, $\overline{T \cap D}$ is closed under addition; hence $f_{1}+f_{2} \in \overline{T \cap D} \subseteq \bar{T}$. The proof that $\bar{T}$ is closed under multiplication follows again from the fact that $u \mapsto r u$ is continuous for all $r \in E$. (See the proof of Lemma 1)

Lemma 10. Let $D \subseteq \mathbb{R}[\underline{X}] \backslash\{0\}$ be a multiplicative set containing 1 . Then a preordering $T$ in $E=D^{-1} \mathbb{R}[\underline{X}]$ is closed if and only if $T \cap \mathbb{R}[\underline{X}]$ is closed in $\mathbb{R}[\underline{X}]$.

Proof. One direction is obvious. Therefore, assume that $T \cap \mathbb{R}[\underline{X}]$ is closed in $\mathbb{R}[\underline{X}]$. Let $V$ be a finite-dimensional subspace of $E$. We claim that $V \cap T$ is closed. Pick a nonzero square $d \in D$ such that $d V \subseteq \mathbb{R}[\underline{X}]$. Then

$$
d(V \cap T)=d V \cap d T=d V \cap T .
$$

The first equality follows from the fact that the mapping $u \mapsto d u$ is bijective on $E$, and the second follows from $T=d T$ ( $d$ is an invertible square in $E$ ). Since $T \cap \mathbb{R}[\underline{X}]$ is closed and $d V \subseteq \mathbb{R}[\underline{X}], d(V \cap T)$ is closed in $d V$. As the mapping $u \mapsto d u$ is a homeomorphism by (F4), it follows that the set $V \cap T$ is closed in $V$. As $V$ was arbitrary, $T$ is closed in $E$.

The next lemmas are needed in the proof of the main theorem of this section. See [4, Chapter 2] for general notions such as the semialgebraic set, semialgebraic homeomorphism, semialgebraic dimension, etc.

Lemma 11. Let $K \subseteq \mathbb{R}^{n}$ be a semialgebraic set. Then there are finitely many semialgebraic subsets $C_{1}, \ldots, C_{s}$ of $K$ such that

(i) $K=C_{1} \cup \ldots \cup C_{s}$,

(ii) each $C_{i}$ is semialgebraically homeomorphic to some $\mathbb{R}^{d_{i}}$,

(iii) each $C_{i}$ has irreducible Zariski closure.

Proof. The proof is by induction on the semialgebraic dimension of $K$. If $\operatorname{dim}(K)=$ 0 , the result is clear, as $K$ is a finite union of points. Hence assume $\operatorname{dim}(K)=$ $m$, and the result is true for all semialgebraic sets of smaller dimension. Let $V$ be the Zariski closure of $K$ and $V_{1}, \ldots, V_{r}$ be its irreducible components. We consider without loss of generality only $K_{1}:=K \cap V_{1}$. The usual semialgebraic cell decomposition allows us to write

$$
K_{1}=\tilde{C}_{1} \cup \ldots \cup \tilde{C}_{k},
$$

where each $\tilde{C}_{j}$ is a semialgebraic set and semialgebraically homeomorphic to some $\mathbb{R}^{d_{j}}$. Now if a $\tilde{C}_{j}$ has the same dimension as $V_{1}$, its Zariski closure equals $V_{1}$, due to the irreducibility of $V_{1}$. Therefore, we use all these $C_{j}$ for our desired decomposition of $K$. The union of all lower-dimensional $C_{j}$ is a semialgebraic set of strictly smaller dimension than $m$, so we can apply the induction hypothesis to it. This yields the result.

Lemma 12. For every semialgebraic set $K$ in $\mathbb{R}^{n}$ and every nonempty multiplicative subset $D$ of $\mathbb{R}[\underline{X}]$, there exists an element $d_{K} \in D$ such that $\overline{K \backslash \mathcal{Z}\left(d_{K}\right)} \subseteq$ $\overline{K \backslash \mathcal{Z}(d)}$ for every $d \in D$. 
Proof. Write $K=C_{1} \cup \ldots \cup C_{s}$ with the properties from Lemma 11 For each $i \in$ $\{1, \ldots, s\}$ and $d \in D$ there are two possibilities. First, $\operatorname{dim}\left(C_{i} \cap \mathcal{Z}(d)\right)<\operatorname{dim}\left(C_{i}\right)$. Since semialgebraic homeomorphisms respect dimensions (4, Theorem 2.8.8]), we see that $C_{i} \cap \mathcal{Z}(d)$ has a (relative) empty interior in $C_{i}$, so $\overline{C_{i} \backslash \mathcal{Z}(d)}=\overline{C_{i}}$. The second possibility is $\operatorname{dim}\left(C_{i} \cap \mathcal{Z}(d)\right)=\operatorname{dim}\left(C_{i}\right)$. Since the Zariski closure of $C_{i}$ is irreducible, it equals the Zariski closure of $C_{i} \cap \mathcal{Z}(d)$. But this means $d$ must vanish on the whole of $C_{i}$, so $\overline{C_{i} \backslash \mathcal{Z}(d)}=\emptyset$. We have proved that for every $d \in D$,

$$
\overline{K \backslash \mathcal{Z}(d)}=\bigcup_{i=1}^{s} \overline{C_{i} \backslash \mathcal{Z}(d)}=\bigcup_{i \in I(d)} \overline{C_{i}},
$$

where $I(d)=\left\{i \mid \operatorname{dim}\left(C_{i} \cap \mathcal{Z}(d)\right)<\operatorname{dim}\left(C_{i}\right)\right\}$. There exist finitely many $d_{1}, \ldots, d_{t} \in$ $D$ such that $\bigcap_{d \in D} I(d)=\bigcap_{j=1}^{t} I\left(d_{j}\right)$. Set $d_{K}:=\prod_{j=1}^{t} d_{j}$ and note that $I\left(d_{K}\right)=$ $\bigcap_{j=1}^{t} I\left(d_{j}\right)$. It follows that for every $d \in D$,

$$
\overline{K \backslash \mathcal{Z}\left(d_{K}\right)}=\bigcup_{i \in I\left(d_{K}\right)} \overline{C_{i}} \subseteq \bigcup_{i \in I(d)} \overline{C_{i}}=\overline{K \backslash \mathcal{Z}(d)}
$$

Lemma 13. For any semialgebraic set $K \subseteq \mathbb{R}^{n}$, the preordering

$$
\operatorname{Pos}^{E}(K)=\left\{R \in D^{-1} \mathbb{R}[\underline{X}] \mid R \succeq 0 \text { on } K\right\}
$$

is closed in $\mathcal{T}_{\text {fin }}$.

Proof. Lemma 12 implies that the set $\mathcal{X}=\overline{K \backslash \mathcal{Z}\left(d_{K}\right)}$ satisfies the assumptions of Lemma 2, hence $\operatorname{Pos}^{E}(\mathcal{X}) \cap \mathbb{R}[\underline{X}]=\operatorname{Pos}(\mathcal{X})$. The set $\operatorname{Pos}(\mathcal{X})$ is closed in $\mathbb{R}[\underline{X}]$, as evaluations in points are linear and therefore continuous. So $\operatorname{Pos}^{E}(\mathcal{X})$ is closed by Lemma 10.

We also have that $\operatorname{Pos}^{E}(\mathcal{X})=\operatorname{Pos}^{E}(K)$. Namely, if $R=\frac{f}{d}$ with $f \in \mathbb{R}[\underline{X}]$ and if $d \in D$ such that $f d \in \operatorname{Pos}\left(K \backslash \mathcal{Z}\left(d_{K}\right)\right)$, then also $R=\frac{f d_{K}}{d d_{K}}$ with $\left(f d_{K}\right)\left(d d_{K}\right) \in$ $\operatorname{Pos}(K)$. The opposite inclusion is trivial.

The following main theorem of this section is an analogue of Theorem 1 above.

Theorem 4. Let $D \subseteq \mathbb{R}[\underline{X}] \backslash\{0\}$ be a multiplicative set containing 1 and $S \subseteq \mathbb{R}[\underline{X}]$ be finite. Suppose there is $p \in D$ with $p \geq 1$ on $K_{S}$ and $k p-\sum_{i=1}^{n} X_{i}^{2} \geq 0$ on $K_{S}$ for some $k \geq 1$. Then in the topology $\mathcal{T}_{\text {fin }}$ on $D^{-1} \mathbb{R}[\underline{X}]$ we have

$$
\overline{T_{S}^{E}}=\operatorname{Pos}^{E}\left(K_{S}\right) \text {. }
$$

Proof. First take $R \in \operatorname{Pos}^{E}\left(K_{S}\right)$, so $R=\frac{f}{d}$ for some $f \in \mathbb{R}[\underline{X}], d \in D$, with $f d \geq 0$ on $K_{S}$. We apply [22, Theorem 5.1] and find $f d$ lying in the closure of $T_{S}^{E}$ in a finite-dimensional subspace of $D^{-1} \mathbb{R}[\underline{X}]$. Hence $f d$, and therefore $R$ belongs to $\overline{T_{S}^{E}}$.

Since $T_{S}^{E} \subseteq \operatorname{Pos}^{E}\left(K_{S}\right) \subseteq \overline{T_{S}^{E}}$, it remains to show that $\operatorname{Pos}^{E}\left(K_{S}\right)$ is closed in $\mathcal{T}_{\text {fin }}$. This is Lemma 13 ,

Remark. We can also deduce Theorem 4 from Theorem 1 and Lemma 13 Namely,

$$
\bigcup_{d \in D} \operatorname{Pos}^{\mathbb{R}[\underline{X}]_{d}}\left(K_{S}\right) \subseteq \bigcup_{d \in D} \operatorname{Pos}^{\mathbb{R}[\underline{X}]_{p d}}\left(K_{S}\right) \subseteq \bigcup_{d \in D} \overline{T_{S}^{\mathbb{R}[\underline{X}]_{p d}}} \subseteq \bigcup_{d \in D} \overline{T_{S}^{\mathbb{R}[\underline{X}]_{d}}}
$$


by application of Theorem 1 to the algebra $\mathbb{R}[\underline{X}]_{p d}$. We use the fact that $\mathcal{T}_{\omega}$ and $\mathcal{T}_{\text {fin }}$ coincide in the countable-dimensional case. Now, using Lemma 13,

$$
\overline{T_{S}^{E}} \subseteq \operatorname{Pos}^{E}\left(K_{S}\right) \subseteq \bigcup_{d \in D} \operatorname{Pos}^{\mathbb{R}[\underline{X}]_{d}}\left(K_{S}\right) \subseteq \bigcup_{d \in D} \overline{T_{S}^{\mathbb{R}[X]}} \subseteq \overline{T_{S}^{E}}
$$

The following is a counterpart to Corollary 1 .

Corollary 2. $\sum \mathbb{R}(\underline{X})^{2}$ is closed in $\mathbb{R}(\underline{X})$ with respect to $\mathcal{T}_{\text {fin }}$.

Proof. Every globally nonnegative rational function is a sum of squares of rational functions.

Example. In $\mathbb{R}(\underline{X} ; \emptyset)$, the sums of squares are not closed. It has been long known that there are nonnegative polynomials which cannot be written as a sum of squares of rational functions without poles. See for example [20, Lemma 1.1] or [18, Paragraph 6]. But as we have seen, these polynomials belong to the closure of the sums of squares in $\mathbb{R}(\underline{X} ; \emptyset)$.

\section{Extension to Finitely Generated $\mathbb{R}$-ALGEBRAS}

The aim of this section is to extend Theorems 1, 2 and 4 from polynomial rings to finitely generated $\mathbb{R}$-algebras.

Let $A$ be a finitely generated $\mathbb{R}$ algebra. A character of $A$ is a unital $\mathbb{R}$-algebra homomorphism from $A$ to $\mathbb{R}$. Write $V(A)$ for the set of all characters of $A$. Elements from $A$ define functions on $V(A)$ by $a(\chi):=\chi(a)$. We equip $V(A)$ with the coarsest topology making all these functions continuous. One can embed $V(A)$ into some $\mathbb{R}^{n}$ by choosing generators $x_{1}, \ldots, x_{n}$ of $A$ and sending $\chi \mapsto\left(\chi\left(x_{1}\right), \ldots, \chi\left(x_{n}\right)\right)$. In that case, the topology on $V(A)$ coincides with the usual topology from $\mathbb{R}^{n}$ (see for example [11, Section 5.7]).

Let $D$ be a multiplicative subset of $A \backslash\{0\}$ which contains 1 . There is a canonical homomorphism $\iota: A \rightarrow F=D^{-1} A$ which, however, is not necessarily one-to-one. For every $d \in A$ write $\mathcal{Z}_{A}(d)=\{\chi \in V(A) \mid \chi(d)=0\}$. Note that the set $V(A) \backslash \bigcup_{d \in D} \mathcal{Z}_{A}(d)$ consists of all characters of $A$ which can be extended (via $\iota$ ) to a character of $F$.

Let $S$ be a finite subset of $A$. Write $K_{S}^{A}=\{\chi \in V(A) \mid \chi(S) \geq 0\}$. Let $T_{S}^{F}$ be the preordering in $F$ generated by the set $\iota(S)$.

For every subset $\mathcal{X}$ of $V(A)$ write $\operatorname{Pos}(\mathcal{X})$ for the set of all $a \in A$ such that $\chi(a) \geq 0$ for every $\chi \in \mathcal{X}$ and write $\operatorname{Pos}^{F}(\mathcal{X})$ for the set of all elements $R \in F$ which have a representation $R=\frac{a}{d}$ with $a \in A, d \in D$ and $a d \in \operatorname{Pos}(\mathcal{X})$.

Theorem 5. Assume that $A, D, S, F$ are as above. Also assume that there is an element $p \in D$ such that $p-1 \in \operatorname{Pos}\left(K_{S}^{A}\right)$ and $k p-\sum_{i=1}^{n} x_{i}^{2} \in \operatorname{Pos}\left(K_{S}^{A}\right)$ for some integer $k \geq 1$ and generators $x_{1}, \ldots, x_{n}$ of $A$. Then:

(1) The closure of $T_{S}^{F}$ in $\left(F, \mathcal{T}_{\omega}\right)$ is $\operatorname{Pos}^{F}\left(K_{S}^{A} \backslash \bigcup_{d \in D} \mathcal{Z}_{A}(d)\right)$.

(2) The closure of $T_{S}^{F}$ in $\left(F, \mathcal{T}_{\text {fin }}\right)$ is $\operatorname{Pos}^{F}\left(K_{S}^{A}\right)$.

(3) For every linear functional $L$ on $F$ such that $L\left(T_{S}^{F}\right) \geq 0$, there exists a measure $\mu$ on $\overline{K_{S}^{A} \backslash \bigcup_{d \in D} \mathcal{Z}_{A}(d)}$ such that

$$
L\left(\frac{f}{d}\right)=\int \frac{f}{d} d \mu \quad \text { for every } \quad \frac{f}{d} \in F
$$


Note that the set $\operatorname{Pos}^{F}\left(K_{S}^{A} \backslash \bigcup_{d \in D} \mathcal{Z}_{A}(d)\right)$ consists of all $R \in F$ such that $\tau(R) \geq 0$ for every character $\tau$ of $F$ which satisfies $\tau(\iota(S)) \geq 0$.

Proof. Let $\pi: \mathbb{R}[\underline{X}] \rightarrow A$ be the unital $\mathbb{R}$-algebra homomorphism defined by $X_{i} \rightarrow$ $x_{i}$ for $i=1, \ldots, n$ and let $\pi^{*}: V(A) \rightarrow \mathbb{R}^{n}$ be the corresponding embedding defined by $\chi \mapsto\left(\chi\left(x_{1}\right), \ldots, \chi\left(x_{n}\right)\right)$. If $f_{1}, \ldots, f_{t}$ are generators of the ideal $I=\operatorname{ker} \pi$, then $\pi^{*}(V(A))=K_{\left\{ \pm f_{1}, \ldots, \pm f_{t}\right\}}$. Similarly, for a given set $S=\left\{g_{1}, \ldots, g_{m}\right\} \subseteq A$ take elements $\tilde{g}_{i} \in \mathbb{R}[\underline{X}]$ such that $\pi\left(\tilde{g}_{i}\right)=g_{i}$ and note that

$$
\pi^{*}\left(K_{S}^{A}\right)=K_{\widetilde{S}}, \quad \text { where } \quad \widetilde{S}:=\left\{\tilde{g}_{1}, \ldots, \tilde{g}_{m}, \pm \tilde{f}_{1}, \ldots, \pm \tilde{f}_{t}\right\} .
$$

Let $\widetilde{D}:=\pi^{-1}(D)$ denote the localization $\widetilde{D}^{-1} \mathbb{R}[\underline{X}]$ by $E$. $\pi$ extends uniquely to a homomorphism $\tilde{\pi}: E \rightarrow F$, making the following diagram commutative:

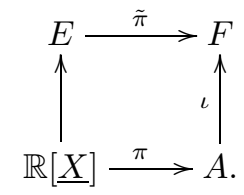

The preorderings $T_{\widetilde{S}} \subseteq \mathbb{R}[\underline{X}]$ and $T_{\widetilde{S}}^{E} \subseteq E$ do not depend on the specific choice of the $\tilde{f}_{i}, \tilde{g}_{i}$ but only on $I$ and $S$. We have

$$
\tilde{\pi}\left(T_{\widetilde{S}}^{E}\right)=T_{S}^{F}
$$

Now suppose $D$ contains an element $p$ with the properties required in Theorem 5 , Any preimage $\tilde{p}$ of $p$ under $\pi$ will have the corresponding property with respect to $K_{\widetilde{S}}$.

For any linear functional $L$ on $F$ with $L\left(T_{S}^{F}\right) \geq 0$, the functional $\widetilde{L}:=L \circ \tilde{\pi}$ fulfills $\widetilde{L}\left(T_{\widetilde{S}}^{E}\right) \geq 0$, and it is therefore an integration on $\overline{K_{\widetilde{S}} \backslash \bigcup_{\tilde{d} \in \widetilde{D}} \mathcal{Z}(\tilde{d})}$ by Theorem 2 , It follows that $L$ is an integration on

$$
\left.\left.\overline{K_{S}^{A} \backslash \bigcup_{d \in D} \mathcal{Z}(d)}\right)=\left(\pi^{*}\right)^{-1} \overline{\left(K_{\widetilde{S}} \backslash \bigcup_{\tilde{d} \in \widetilde{D}} \mathcal{Z}(\tilde{d})\right.}\right) .
$$

Therefore, we have proved assertion (3) of Theorem 5 . Assertions (1) and (2) of Theorem [5 follow from Theorems 1 and 4 , observations

$$
\begin{aligned}
& \tilde{\pi}\left(\operatorname{Pos}^{E}\left(K_{\widetilde{S}} \backslash \bigcup_{\tilde{d} \in \widetilde{D}} \mathcal{Z}(\tilde{d})\right)\right)=\operatorname{Pos}^{F}\left(K_{S}^{A} \backslash \bigcup_{d \in D} \mathcal{Z}_{A}(d)\right), \\
& \tilde{\pi}\left(\operatorname{Pos}^{E}\left(K_{\widetilde{S}}\right)\right)=\operatorname{Pos}^{F}\left(K_{S}^{A}\right)
\end{aligned}
$$

and $(*)$ and from the following lemma.

Lemma 14. Let $E$ and $F$ be $\mathbb{R}$-vector spaces, $\tilde{\pi}: E \rightarrow F$ a linear mapping which is onto and $C$ a convex cone in $E$ which contains $\operatorname{ker} \tilde{\pi}$. Then

$$
\tilde{\pi}(\bar{C})=\overline{\tilde{\pi}(C)},
$$

where either $E, F$ are both equipped with $\mathcal{T}_{\omega}$ or both with $\mathcal{T}_{\text {fin }}$.

Proof. Suppose that $E, F$ are equipped with $\mathcal{T}_{\omega}$. The inclusion $\tilde{\pi}(\bar{C}) \subseteq \overline{\tilde{\pi}(C)}$ follows from the fact that $\tilde{\pi}$ is continuous in $\mathcal{T}_{\omega}$. To prove the opposite inclusion, pick $\tilde{\pi}(e) \in \overline{\tilde{\pi}(C)}$. Then $L(\tilde{\pi}(e)) \geq 0$ for every linear functional $L$ on $F$ such that $L(\tilde{\pi}(C)) \geq 0$. Every linear functional $L^{\prime}$ on $E$ such that $L^{\prime}(C) \geq 0$ factors through 
$\tilde{\pi}$ because ker $\tilde{\pi} \subseteq C$. It follows that $L^{\prime}(e) \geq 0$ for every linear functional $L^{\prime}$ on $E$ such that $L^{\prime}(C) \geq 0$. Therefore, $e \in \bar{C}$ which implies that $\tilde{\pi}(e) \in \tilde{\pi}(\bar{C})$.

Now suppose that $E, F$ are equipped with $\mathcal{T}_{\text {fin }}$. First note that by the same argument as in Lemma 9, the closure of a convex cone with respect to $\mathcal{T}_{\text {fin }}$ is again a convex cone. Now the formula $\tilde{\pi}(\bar{C}) \subseteq \overline{\tilde{\pi}(C)}$ follows from the fact that $\tilde{\pi}$ is continuous. To prove the opposite inclusion it suffices to show that $\tilde{\pi}(C)$ is closed for every closed cone $C$ containing ker $\tilde{\pi}$. Suppose that $C$ is such a cone and pick a finite-dimensional subspace $W$ of $F$. Let $V$ be a finite-dimensional subspace of $E$ such that $\tilde{\pi}(V)=W$. By assumption, $C \cap V$ is closed in $V$ in the Euclidean topology, hence also in the finest locally convex topology. Since $C \cap V$ is a closed cone in $V$ which contains $V \cap \operatorname{ker} \tilde{\pi}=\left.\operatorname{ker} \tilde{\pi}\right|_{V}$, it follows by the first paragraph (applied to $\left.\tilde{\pi}\right|_{V}: V \rightarrow W$ instead of $\left.\tilde{\pi}: E \rightarrow F\right)$ that $\tilde{\pi}(C \cap V)$ is closed in $W=\tilde{\pi}(V)$. It remains to show that $\tilde{\pi}(C \cap V)=\tilde{\pi}(C) \cap \tilde{\pi}(V)$. Pick $\tilde{\pi}(e) \in \tilde{\pi}(C) \cap \tilde{\pi}(V)$. We have that $e=c+i=v+j$ for some $c \in C, v \in V$ and $i, j \in \operatorname{ker} \tilde{\pi}$. Since $\operatorname{ker} \tilde{\pi} \subseteq C$, $c+i-j=v$ belongs to $C \cap V$. It follows that $\tilde{\pi}(e)=\tilde{\pi}(v)$ belongs to $\tilde{\pi}(C \cap V)$. The opposite inclusion is clear.

It would be interesting to know whether Theorem 5 also holds for the algebra $A=\mathcal{O}\left(\mathbb{R}^{n}\right)$ of analytic functions on $\mathbb{R}^{n}$. In this case we can use [1, Theorem 2.4] instead of [22, Theorem 5.1], so the assumption $p \in D$ for certain $p$ may not be necessary.

\section{REFERENCES}

[1] F. Acquistapace, C. Andradas, F. Broglia, The strict Positivstellensatz for global analytic functions and the moment problem for semianalytic sets, Math. Ann. 316 (2000), pp. 609616. MR 1758445 (2001g:14087)

[2] N. I. Akhiezer, The Classical Moment Problem and Some Related Questions in Analysis, Oliver \& Boyd, Edinburgh/London, 1965. MR0184042 (32:518)

[3] T. M. Bisgaard, The topology of finitely open sets is not a vector space topology, Arch. Math. 60 (1993), pp. 546-552. MR.1216700 (94g:46013)

[4] J. Bochnak, M. Coste, M.-F. Roy, Real Algebraic Geometry, Erg. Math. Grenzgeb. 36, Springer, Berlin, 1998. MR.1659509 (2000a:14067)

[5] N. Bourbaki, Topological vector spaces, Chapters 1-5, English edition, Springer Verlag, Masson, 1987. MR0910295 (88g:46002)

[6] A. Bultheel, P. González-Vera, E. Hendriksen, O. Njåstad, Orthogonal rational functions, Cambridge Monographs on Applied and Computational Mathematics vol. 5, Cambridge University Press, Cambridge, 1999. MR1676258(2000c:33001)

[7] A. Bultheel, P. González-Vera, E. Hendriksen, O. Njåstad, Elements of a theory of orthogonal rational functions, Rev. Acad. Canaria Cienc. 11 (1999), pp. 127-152. MR1780786 (2001i:42041)

[8] J. D. Chandler, Jr., Rational moment problems for compact sets, J. Approximation Theory 79 (1994), pp. 72-88. MR1294321 (95h:44019)

[9] S. Kuhlmann, M. Marshall, Positivity, sums of squares and the multidimensional moment problem, Trans. Amer. Math. Soc. 354 (2002), pp. 4285-4301. MR1926876 (2003j:14078)

[10] S. G. Krantz, H. R. Parks, Geometric Integration Theory, http://www.math. wustl.edu/ $\sim$ sk/books/root.pdf.

[11] M. Marshall, Positive polynomials and sums of squares, AMS Surveys and Monographs vol. 146, AMS, Providence, 2008. MR 2383959

[12] M. Marshall, Extending the Archimedean Positivstellensatz to the non-compact case, Canad. Math. Bull. 44 (2001), pp. 223-230. MR1827856 (2002b:14073)

[13] M. Marshall, Approximating positive polynomials using sums of squares, Canad. Math. Bull., 46 (2003), pp. 400-418. MR.1994866 (2004f:14084) 
[14] T. W. Palmer, Banach algebras and the general theory of $*$-algebras, Vol. 2, Encyclopedia of Mathematics and its Applications, 79. Cambridge University Press, Cambridge, 2001. pp. i-xii and 795-1617. MR.1819503 (2002e:46002)

[15] A. Prestel, C. N. Delzell, Positive polynomials, Springer, Berlin, 2001. MR.1829790 (2002k:13044)

[16] M. Putinar, F.-H. Vasilescu, Solving moment problems by dimensional extension, Ann. of Math. (2) 149 (1999), pp. 1087-1107. MR.1709313(2001c:47023b)

[17] B. Reznick, Uniform denominators in Hilbert's Seventeenth Problem, Math. Z. 220 (1995), pp. 75-97. MR 1347159 (96e:11056)

[18] B. Reznick, Some concrete aspects of Hilbert's 17th problem, in: Real Algebraic Geometry and Ordered Structures (C. N. Delzell, J.J. Madden eds.), Cont. Math., vol. 253 (2000), pp. 251-272. MR1747589 (2001i:11042)

[19] W. Rudin, Real and Complex Analysis, 3rd ed., McGraw-Hill, 1987. MR0924157 (88k:00002)

[20] C. Scheiderer, Sums of squares of regular functions on real algebraic varieties, Trans. Am. Math. Soc. 352 (1999), pp. 1039-1069. MR1675230 (2000j:14090)

[21] K. Schmüdgen, The K-moment problem for compact semi-algebraic sets, Math. Ann. 289 (1991), pp. 203-206. MR.1092173 (92b:44011)

[22] M. Schweighofer, Iterated Rings of Bounded Elements and Generalizations of Schmüdgen's Positivstellensatz, J. Reine Angew. Math. 554 (2003), pp. 19-45. MR.1952167 (2004b:13028) (unpublished Erratum available at http://perso.univ-rennes1. $\mathrm{fr} /$ markus.schweighofer/publications/irobeerr.pdf)

[23] J. Stochel, F. H. Szafraniec, Algebraic operators and moments on algebraic sets, Portugal. Math. 51 (1994), 25-45. MR 1281954 (95d:47013)

[24] F.-H. Vasilescu, Spaces of fractions and positive functionals, Math. Scand. 96 (2005), 257279. MR2153414 (2007b:47040)

[25] J. H. Williamson, On topologizing the field $\mathbb{C}(t)$, Proc. Amer. Math. Soc. 5 (1954), pp. 729734. MR0063574(16:145f)

Faculty of Mathematics and Physics, University of Ljubljana, Jadranska 21, SI-1000 Ljubljana, SloveniJa

E-mail address: cimpric@fmf.uni-lj.si

Department of Mathematics and Statistics, University of Saskatchewan, Saskatoon, Saskatchewan, Canada S7N 5E6

E-mail address: marshall@math.usask.ca

Fachbereich Mathematik und Informatik, Universität Leipzig, D-04009 Leipzig, GerMANY

E-mail address: tim.netzer@math.uni-leipzig.de 